\title{
CRISPR-VAE: A Method for Explaining CRISPR/Cas12a Predictions, and an Efficiency-aware gRNA Sequence Generator
}

\author{
Ahmad Obeid, and Hasan Al-Marzouqi
}

\begin{abstract}
-
Motivation: Sizeable research has been conducted to facilitate the usage of CRISPR-Cas systems in genome editing, in which deep learning-based methods among others have shown great promise in the prediction of the gRNA efficiency. An accurate prediction of gRNA efficiency helps practitioners optimize their engineered gRNAs, maximizing the on-target efficiency, and minimizing the off-target effects. However, the black box prediction of deep learning-based methods does not provide adequate explanation to the factors that make a sequence efficient; rectifying this issue can help promote the usage of CRISPR-Cas systems in numerous domains.

Results: We put forward a framework for interpreting gRNA efficiency prediction, dubbed CRISPR-VAE, and apply it to CRISPR/Cpf1. We thus help open the door to a better interpretability of the factors that make a certain gRNA efficient. We further lay out a semantic articulation of such factors into position-wise k-mer rules. The paradigm consists of building an efficiency-aware gRNA sequence generator trained on available real data, and using it to generate a large amount of synthetic sequences with favorable traits, upon which the explanation of the gRNA prediction is based. CRISPR-VAE can further be used as a standalone sequence generator, where the user has access to a low-level editing control. The framework can be readily integrated with different CRISPR-Cas tools and datasets, and its efficacy is confirmed in this paper.

Availability and implementation: The source code will be shared publicly upon acceptance.

Contact: ahmad.obeid@ku.ac.ae
\end{abstract}

Index Terms-CRISPR, Explainable deep learning

\section{INTRODUCTION}

$\mathbf{T}$ HE usage of CRISPR-Cas systems for genome editing has been gaining much popularity recently due to the many applications which the technology enables in various domains such as gene therapy and agricultural engineering [1]-[4]. Such popularity motivated an advancement in the related research, particularly including many works towards the prediction of guide RNAs (gRNAs) efficiency. In CRSIPR-Cas systems, gRNAs locate DNA targets for the endonuclease to cleave. When the DNA strands are being repaired, the process results in random insertions/deletions or precise gene editing that can be exploited for gene knockins [5]. The efficacy of said process is thus a function of the used gRNA; predicting it is important for a safe usage of CRISPR-Cas systems, in order to ensure high on-target indel efficacy, and minimum off-target effects. Additionally, the discovery of Cas12a, also

The authors are with the Department of Electrical Engineering and Computer Science at Khalifa University, Abu Dhabi, UAE.

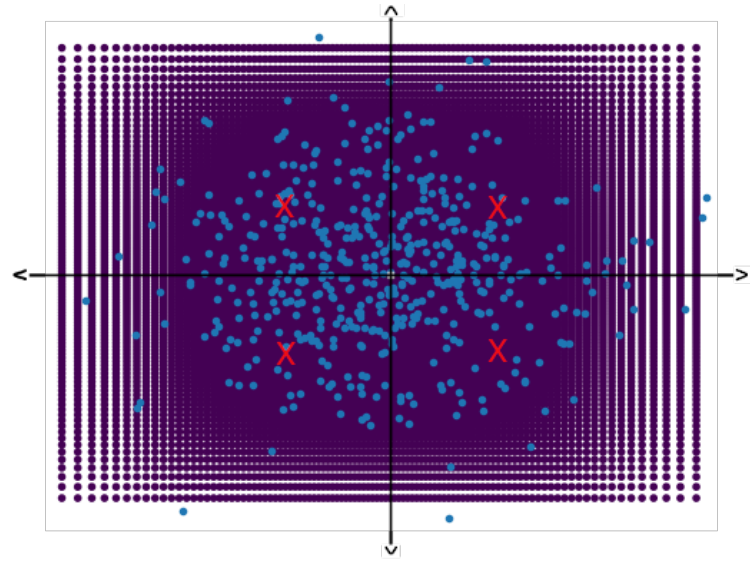

Fig. 1. The latent space obtained by CRISPR-VAE, showing the seeds of the synthetic data (dark points) bridging sequence-related gaps left by publicly available data (light points). The cross-marks indicate the quadrant centroid

known as CRISPR from Prevotella and Francisella1 (Cpf1) as an alternative endonuclease to CRISPR associated protein 9 (Cas9) in the CRISPR systems is an important development, and introduces many favorable features. For example, Cpf1 is shorter in size, requires a smaller CRISPR Ribonucleic Acid (RNA) to function, and facilitates the re-engineering of the desired DNA as the target, with the Protospacer Adjacent Motif (PAM) remaining unaffected [6]. Additionally, Cpf1 has shown better specificity in human and plant cells than Cas9, and enables the editing of Corynebacterium glutamicum and Cyanobacteria, which was not possible with Cas9 [5].

The methods used for gRNA efficiency prediction can be alignment-based, hypothesis-driven, or learning-based [7]. Alignment-based methods rely entirely on locating the PAM, in order to align the gRNA in the genome. Hypothesisdriven methods score the aligned gRNAs by other contextual factors. Learning-based methods train a prediction model that can extract many hidden sequence-related factors. With the continuous advancement in the area of deep learning, learningbased methods have been showing high accuracy and a promising performance at gRNA efficacy prediction. However, these methods are still inadequately interpreted, and provide little explainability to their predictions. Said explainability is crucial for a better understanding of CRISPR systems, and to explore the factors that make certain gRNAs lead to higher on-target activities, and lower off-target effects. This enables practitioners design better sequences, and analysts 
better diagnose their models' decisions, which promotes the application of genome editing in different domains.

Previous attempts have touched upon this research direction [5], [7]-[9], but were faced with the two challenges of deficient and incomprehensive data. The deficiency in the data is represented by having relatively few sequences that belong to specific efficiency categories. This, in turn, disallows the establishment of statistically significant factors. As for the incomprehensiveness of the data, we are referring to the case where available datasets consist of an incohesive collection of sequences that exhibit many sequence-related features, where no clear connection can be drawn. This scatters the effort of finding the features that are responsible for high editing efficiency. In this work, we develop a framework that tackles both problems simultaneously, as illustrated in Fig.1. More concretely, publicly available data of CRISPR/Cpf1 activity leaves sequence and structure-related gaps in a certain analysis space (to be explained later), which we place a magnifying lens over; manifested in the development of a sequence generator dubbed CRISPR-Variational Autoencoder (CRISPR-VAE).

CRISPR-VAE is efficiency-aware, and is used to synthesize numerous sequences of high and low efficiencies. These sequences are not arbitrary, but form a structured analysis space that is meant to bridge said gaps left by the dataset, and exhibits different sequence phenomena ordered in different locations in the space. As such, this produces more comprehensive and plentiful data, upon which the discovery of rules is based. Such a paradigm concentrates and magnifies the search for efficiency-promoting factors. Finally, we predict the efficiency of the synthetic sequences using the deep learningbased predictor seq-DeepCpf1 which previously showed good performance on the dataset [10]. As such, we establish an agreement between two methodologically distinct frameworks: a generative one and discriminative one, which increases the confidence in the findings.

In summary, the contribution of our work is:

- putting forward a deep learning-based explainability framework, which can be readily integrated with any CRISPR-Cas dataset;

- developing methods that improve the statistical significance of findings, and concentrates the search for them;

- semantically articulating the high-quality findings of the mentioned methods in a suitable k-mer paradigm;

- developing the standalone sequence generator CRISPRVAE, which can generate sequences of high (or low) efficiencies with low-level, position-wise features tailored to the practitioner's needs;

- demonstrating the correctness of findings by establishing an agreement between a descriminative and a generative method.

In the following, we will provide a literature review in Section II for the related works in the themes of deep learningbased prediction of gRNA efficiency and explainability. In Section III, we will describe how a structured analysis space is obtained, how an efficiency-aware gRNA generator is made, and how the different sequence features are extracted. Finally, we summarize the results of our experimentation, and mention some concluding remarks in Sections IV and V.

\section{RELATED WORK}

The task of predicting a quantifiable quality assessment of a gRNA sequence is realized through predicting its ontarget efficiency and off-target effects. In this vain, the ability to flesh-out well-articulated rules that can be interpreted by humans further helps practitioners decipher the genome code. Consequently, researchers have been developing analytical tools for the mentioned tasks, among which deep learningbased tools have been showing special promise due to the continuous advancement in the field [7], [10]-[14]. In spite of that, the issue of interpretation in deep learning-based tools towards the prediction of gRNA efficiency is still immature, and such methods still lack strong explainability that can guide the analysis in a meaningful fault-diagnosis direction.

In [10], the DeepCpf1 and seq-DeepCpf1 predictors have been developed using Convolutional Neural Networks (CNNs) and dense ones. Said predictors show an improved performance in comparison to other classical methods. In [11], the authors follow a similar path, but the support vector regression (SVR) is used at the end to aid the CNN network, showing some improvements in the performance. Nonetheless, both proposed paradigms, which target the Cpf1 endonuclease, do not provide any explainability for their prediction. Similarly, the DeepCas9 [13] makes use of CNNs, DeepCRISPR [12] aids them with an Autoencoder (AE) stage for unsupervised representation learning, and the C-RNNCrispr [7] aids them with a Recurrent Neural Network (RNN) for a better sequence learning. All these methods and others have been developed for the cas9 endonuclease. Moreover, DeepPE [14] was introduced for the new tool of Prime Editing, also making use of CNNs. Despite the versatility and prowess introduced to tackle the task of gRNA efficiency prediction, most of such paradigms do not tackle the issue of explainability, and deal with their predictors as black boxes. Additionally, there are various works that employ deep learning for gRNA off-target prediction [15][17], also suffering from the black-box behaviour of their predictors.

On the other hand, the issue of explainability in deep learning-based prediction of gRNA efficiency has been studied. For example, [7] studies an optimization of their model score with respect to the inputted gRNA sequence, in order to infer the most prominent features that maximize the efficiency. A similar approach is also used in [18]. Contrariwise, some works opt for classical machine learning tools that are easier to explain [19], thus trading-off accuracy with explainablity.

Other attempts that employ statistical analysis of the available data to infer position-wise base preference rules [5], [8], [9] are relevant, although they do not employ deep learningbased methods for their rule inference. However, the main issue with such methods is that they base the analysis exclusively on the available data, which exhibits a few limitations. Firstly, the available data is limited in quantity (i.e. classwise), despite publishing large datasets for both cas 9 and $\mathrm{Cpf} 1$ endonucleases. For example, the available data on Cpf1 has a small number of sequences with efficiency $\geq 0.99$ or $\leq 0.05$, although having a large number of such greatly polarized sequences is important to infer the most prominent rules 


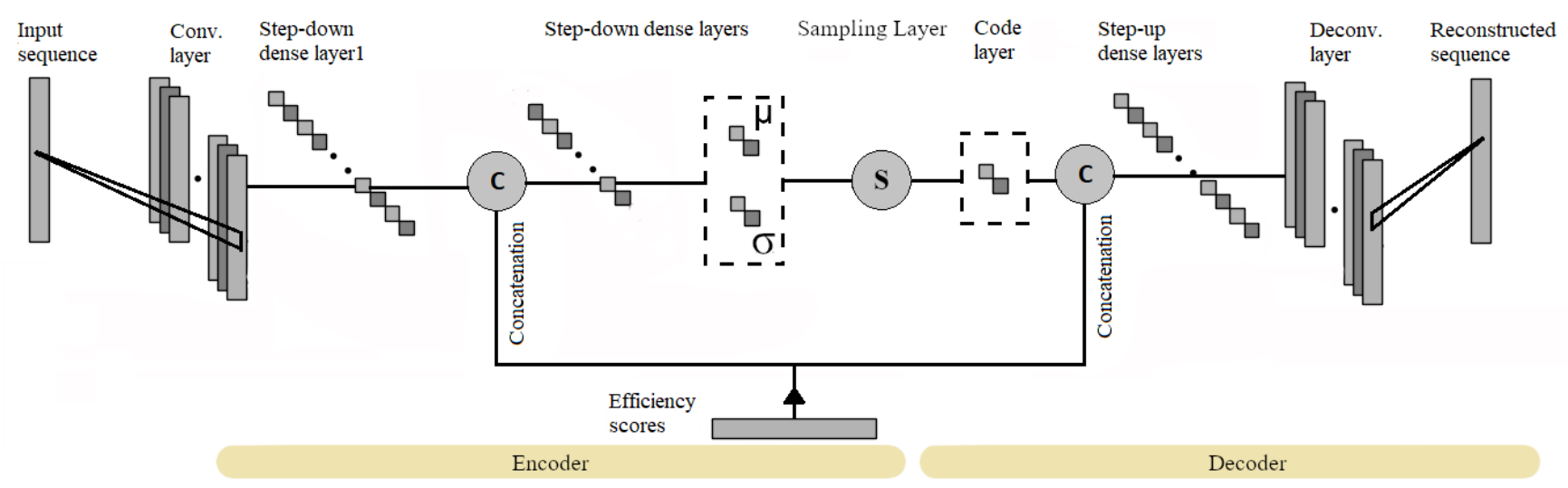

Fig. 2. CRISPR-VAE architecture, shown to integrate the efficiency information at two stages: end of encoder, and beginning of decoder

to a statistically significant degree [20], [21]. More gravely, the available data suffers from qualitative limitation. In other words, the available data is usually scattered in a sequencecohesion sense, exhibiting different and distinct sequencerelated features. Each one of these features is also obscurely represented in the data, making it difficult to discover them. Theoretically, a quantitative comprehensiveness cannot be realistically achieved. Instead, we seek to meaningfully expand the data to signify the different features both quantitatively and qualitatively. In this work, we focus on building a framework that is well embedded in the deep learning paradigm, and that can be generically applied to any CRISPR-Cas system, while tackling the mentioned difficulties in the available data.

\section{Materials AND Methods}

In this Section, we will describe the three main components of our proposed framework, which starts with the proposed generative framework CRISPR-VAE and its advantages, then describes the subsequent feature extraction procedure.

\section{A. VAE for a Structured Latent Space}

We start by describing the general paradigm of VAE, which enables establishing a structured latent space, and the benefit of the latter.

The illustration in Fig.1 demonstrates the core of the analysis paradigm. In contrast to previous attempts, the proposed work aims to accentuate and distinguish the existing sequencerelated phenomena, and explore possible ignored ones in their neighborhood. This necessitates establishing a structured analysis space, for which we employ the VAE paradigm. The analysis space is composed of numerous synthetic sequences, that share a resemblance with the training data, and that are systematically distributed over the space.

The generative process of the VAE starts by generating latent variable $\mathbf{z}$ from the prior distribution $p_{\theta}(\mathbf{z})$. Then, $\mathbf{x}$ is generated (reconstructed) from the generative distribution $p_{\theta}(\mathbf{x} \mid \mathbf{z})$. In this framework, parameter estimation is difficult due to the intractability of the posterior. Alternatively, the lower-bound of the log likelihood is used:

$\log \left(p_{\theta}(\mathbf{x})\right) \geq-D_{K L}\left(q_{\phi}(\mathbf{z} \mid \mathbf{x}) \| p_{\theta}(\mathbf{z})\right)+\mathbb{E}_{q_{\phi}(z \mid x)}\left[\log p_{\theta}(\mathbf{x} \mid \mathbf{z})\right]$ where $q_{\phi}(\mathbf{z} \mid \mathbf{x})$ is an approximation for the true posterior $p_{\theta}(\mathbf{z} \mid \mathbf{x})$, and $D_{K L}(. \|$.$) is the KL-divergence. In our imple-$ mentation, we use CNNs and dense layers for the realization of both models $p_{\theta}(\mathbf{x} \mid \mathbf{z})$ and $q_{\phi}(\mathbf{z} \mid \mathbf{x})$ i.e. the encoder and decoder models, respectively, as shown in figure 2. Furthermore, assuming a Gaussian latent variable, the empirical loss of the VAE can be written as:

$$
\mathbb{L}=-D_{K L}\left(q_{\phi}(\mathbf{z} \mid \mathbf{x}) \| p_{\theta}(\mathbf{z})\right)+\frac{1}{L} \sum_{t=1}^{T} \log \left(p_{\theta}\left(\mathbf{x} \mid z^{(t)}\right)\right)
$$

where $z^{(t)}$ is a sample drawn from the generative model i.e. $z^{(t)}=g_{\phi}(\mathbf{x}, \epsilon)$, and $\epsilon \sim \mathcal{N}(0, \mathbf{1})$ is used for the so-called reparametrization trick [22].

The encoder model learns to project the sequences into the latent space, assimilating them into a normal distribution, where different sequence-related features occupy different locations of the space. Nevertheless, the projected sequences of the training data exhibit a non-cohesive latent space, leaving certain gaps for exploration. As such, a systematic and structured sampling from the latent space for decoding will result in the synthesis of novel sequences that resemble the original data, and that fill in the left gaps, which provides a continuous and smooth bridging between the different sequence phenomena.

The shown latent space in Fig. 1 is two-dimensional (2D), but the analysis can be extended to higher dimensions, giving more prowess to the VAE architecture, and thus obtaining a higher quality of reconstruction and synthesis, and a larger amount of synthetic data. This comes over the expense of a more complex analysis and a demand of higher storage capacity.

The benefit of having a structured latent space is two-fold. Firstly, we ensure that all phenomena existing and scattered in the original data are parsed and highlighted. Secondly, it was empirically demonstrated that the structured space systematically distributes the different sequence phenomena in its different locations (e.g., quadrants in 2D space). This eases the analysis and search for efficiency-promoting features. Additionally, this gives the sequence generator a low-level capability of editing, where specific position-wise base preferences are translated to sampling from different quadrants. 


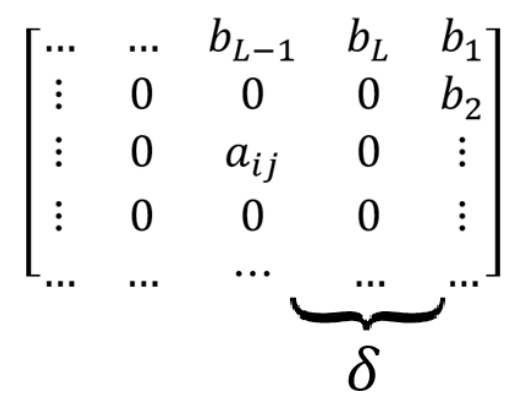

Fig. 3. Filter for generating heat maps, according to equation (3)

Moreover, without a structured latent space for analysis, exploring ignored potential phenomena in a comprehensive manner would be too wasteful and demanding of resources, with an estimated upper-limit complexity in the search space of $\mathcal{O}\left(31^{4}\right)$ (assuming gRNA sequences of length 34, with a known PAM of TTTV), where the vast majority of such sequences would have no connection to the available data, rendering the validation impossible. Instead, the proposed framework enables the synthesis to be confined to sequences that resemble the available data. In our implementation, we sampled 10,000 latent codes arranged in a grid of 100x100 which are decoded to synthetic sequences for subsequent analysis stages.

To test the structure of the latent space, distance heat maps have been constructed through the following:

$$
M a p_{i j}^{k}=\frac{1}{L} \sum_{l=1}^{L} H\left(\hat{a_{i j}}, \hat{b_{l}}\right),\left\{b_{l}: D^{(\infty)}\left(a_{i j}, b_{l}\right)=\delta_{k}\right\}
$$

where $\mathrm{i}$ and $\mathrm{j}$ denote each point in the heat map, $\mathrm{k}$ denotes the specific heat map that corresponds to the used $\delta, \hat{\mathbf{a}}=g_{\phi}(\mathbf{a}, \epsilon)$, $\hat{\mathbf{b}}=g_{\phi}(\mathbf{b}, \epsilon), H$ is the Hamming distance, and $D^{(\infty)}$ is the Minkowski distance of order $\infty$. L denotes the number of seeds in the latent space that satisfy the Minskowski distance condition. A structured space is expected to exhibit heat maps with values growing proportional to $\delta$. For simplicity, the generation of each map can be described as a convolution with the filter in Fig. 3, with the multiplication operation substituted by the Hamming distance, as described in equation (3), and zeros indicating no operation.

\section{B. CVAE for efficiency-awareness}

In our implementation, we specifically follow the conditional VAE (CVAE) paradigm inspired by [22], where we condition on the efficiency score of each sequence. In this Section, we will describe the needed change that grants CRISPR-VAE its efficiency-awareness.

More concretely, equation (2) becomes:

$\mathbb{L}_{\mathbb{C}}=-D_{K L}\left(q_{\phi}(\mathbf{z} \mid \mathbf{x}, \mathbf{c}) \| p_{\theta}(\mathbf{z} \mid \mathbf{c})\right)+\frac{1}{L} \sum_{t=1}^{T} \log \left(p_{\theta}\left(\mathbf{x} \mid z^{(t)}, \mathbf{c}\right)\right)$

where we are conditioning the encoding, the decoding, and the prior distribution on the efficiency information $c$. This means that we obtain a separate latent space for each efficiency category. In our implementation, we convert the efficiency scores in the public data to integers, resulting in a 100 classes $(0-99)$. Theoretically, this results in up to 100 latent spaces, each consisting of $100 \times 100$ grid of sequences. However, we confined the synthesis to classes 0 -efficiency and 99-efficiency to synthesize the most polarized sequences, in order to focus on the most prominent and distinct sequence-related features that set the high-efficiency sequences apart from their lowefficiency counterparts.

Integrating the CVAE paradigm has two benefits. Firstly, the available data provides efficiency measurements which represent useful information to improve the quality of synthesis and reconstruction of the VAE. Indeed, experimentation showed that exploiting the efficiency information improves the performance of the VAE, as will be shown in Section IV. Secondly, exploiting the efficiency information of the data makes the VAE efficiency-aware, and makes the synthesis of the data tailored to the needs of the user (e.g., focused on the high-efficiency sequences). Moreover, this enables us to prob the agreement between the CVAE and existing descriminative methods. In Section IV, we show that CRISPR-VAE and the seq-DeepCpf1 predictor [10] have a strong agreement, and thus increasing the confidence in the findings, without the need for laboratory testing. Herein, another benefit of having a structured latent space is apparent, where we can enforce a resemblance between the synthetic data and the original data, making seq-DeepCpf1 familiar with the synthetic data.

A final trick was employed to improve the quality of reconstruction of CRISPR-VAE. Particularly, the first three loci in the PAM of all sequences were removed, as they are constantly TTT. It was observed upon experimentation that the model finds reconstructing such motif as an easy way to score highly in the objective function; avoiding it motivated the model to rely on learning more interesting sequence-related features that improve the quality of reconstruction, which had a direct impact on said quality.

Fig.2 illustrates the CVAE paradigm. The one-hot-encoded efficiency information is fed to the network at two concatenation stages. The first stage, which comes after the first dense layer, allows the efficiency information to be blended and integrated with the sequence information via the subsequent dense layers, and into the embedding of the code layer, establishing the latent space. The second stage, which comes after the code layer, allows the decoder to be a standalone efficiencyaware sequence generator. The sampling layer employs the aforementioned reparametrization trick to convert $\mu$ and $\sigma$ to the latent codes.

\section{Data usage}

As efficiency scores are used, it is applicable to split our data into training and testing sets to confirm the generality of our findings. We use the data provided in [10], where highthroughput experiments were used to generate sets HT1, HT2, and HT3. We use set HT1 for training, which consists of $\sim 16300$ sequences, while sets HT2 and HT3 were used for testing. These sets do not share any sequences, which excludes 


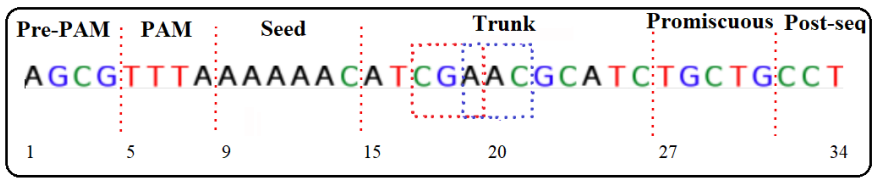

Fig. 4. Different regions in gRNA, with an example of two overlapping mer windows of size 3 in the trunk region

any possibility of data leakage. We also applied data augmentation by causing small perturbations in the promiscuous region of each sequence in HT1 such that the efficiency scores are likely maintained according to [9], resulting in $\sim 85,000$ sequences to train CRISPR-VAE.

\section{Feature Extraction}

Having built CRISPR-VAE, one can synthesize numerous sequences that exhibit the two main characteristics missing from the original data: comprehensiveness and plentifulness. What remains is to extract the sequnce-related features that are responsible for the disparity in the sequences that belong to 0 -efficiency and 99-efficiency classes. To that end, two methods were used to extract and articulate the most prominent features explored in the synthetic data. The first one consists of k-mer histogramming analysis to build Mer Significance Maps (MSMs), and the second consists of visualizing class activation maps (CAMs) [23] produced by a binary classifier that distinguishes the high-efficiency from the low-efficiency sequences.

Firstly, following [10], we define the three regions of seed, trunk, and promiscuous, in addition to PAM, pre-PAM, and post-seq regions, as shown in figure 4 . In the first method, we employ a moving overlapping window encapsulating $\mathrm{k}$-mers to segregate the feature extraction based on the position in the gRNA sequence. This confers the analysis with the needed contextual specificity. We chose the step size to be 1 base, resulting in $L-k+1$ sub-regions for each parent region, where $L$ is the length of the region (e.g., $L_{\text {trunk }}=12$ ), and $k$ is the mer size. In this paper, we constricted the experimentation to $k=3$, but other options can be easily realized. Also, we split the latent space into equal-sized locations (e.g., 4 quadrants in $2 \mathrm{D}$ space), where the histogramming takes place independently. This is to highlight certain phenomena that may otherwise be overshadowed by more prominent ones. It was indeed observed that different sequence features are prominent in different locations in the latent space.

After pooling in all possible features, we filtered them by firstly setting an empirical significance threshold $(\eta)$ where features with frequency below this threshold are discarded of. The threshold is chosen as $m \%$ (typically $7 \sim 10 \%$ ) of the number of possible mers, multiplied by the number of sequences under analysis $(N)$, as shown in equation (5). $N$ can refer to the whole set of generated sequences, or only sequences in a specific quadrant. $\eta$ is defined for each quadrant, for each region in the gRNA, and for high-efficiency and low-efficiency sequences, separately, albeit with a constant $m \%$ throughout. Secondly, we discarded of features that are simultaneously above the low-efficiency and high-efficiency significance thresholds i.e. the common features between the two classes. Moreover, we highlight some novel features that are obscure in the training data, but are discovered due to the mentioned benefits introduced by the synthetic data, and are later found to exist in the testing data. In other words, these features would have likely been ignored if the analysis did not involve synthesizing sequences (i.e. similar to [5]), alluding to the added generality conferred by the proposed framework. We can thus point out the differences between the proposed paradigm and that of [5] as introducing more specificity by exploiting the hidden cohesion in the training data, more sensitivity by highlighting possibly overshadowed features, and more generality by discovering the neighborhood of the real sequences.

$$
\eta=\frac{m}{100}(L-K+1) * N
$$

In the second method, we trained a binary classifier on the synthetic data, and visualized the CAMs [23] for each quadrant separately. Said CAMs are obtained by maximizing the score of the binary efficiency classifier with respect to the first convolutional layer. This results in attention maps that visually describe the reason for the decision of the classifier. We obtained such maps for the decision of all 99efficiency sequences, and averaged them. Such features can be easily compared with their counterparts from the first method, thus enabling testing the agreement between both methods. Moreover, the two methods are complementary to each other. The first method has a finer granularity in terms of the location of the prominent features, while the second is an automatic method that directly explains the decision of the binary classifier. By looking at the results of both methods, numerous features can be extracted, and a better understanding of the composition of an efficient sequence can be made. Finally, we use Welch's t-test to evaluate the statistical significance of the explored features. As such, we include the p-values of observing a high-efficiency sequence, exhibiting each of the explored features.

The full code of the methodology, including all filtering stages, and all generated results is shared publicly. ${ }^{1}$

\section{RESUlts}

We split the Results Section into two parts. In the first one, we show results that confirm the efficacy of the proposed paradigm. Secondly, we summarize the inferred sequencerelated features from the two methods mentioned in III-D. Finally, we explain how CRISPR-VAE can be used as a sequence generator, and the method by which users can control the nucleotide content of their synthetic sequences from the 99-efficiency class.

\section{A. Confirming the Validity of the Proposed Framework}

We start by confirming that the constructed latent space is structured. A structured latent space exhibits smooth transitioning between the different sequences, placing similar sequences in each others' vicinity. As such, when varying

\footnotetext{
${ }^{1}$ will be published upon acceptance
} 


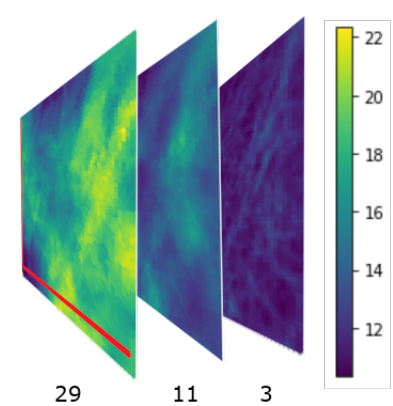

Fig. 5. Results of heat maps generation by equation (3), with $\delta=\{3,11,29\}$

$\delta$ (equation (3), a structured latent space should give heat maps with values growing proportional to it. In Fig. 5, $\delta=\{3,11,29\}$ in order to compare sequences with small, medium and large separations. The shown heat maps are for the class 99-efficiency sequences. Indeed, the heat maps behave as expected from a structured space, with the smallest values observed with $\delta=3$, and the largest with $\delta=29$.

Afterwards, in table I, we show that the utilization of the efficiency scores is beneficial to the reconstruction quality of the CRISPR-VAE. Eventually, this quality is reflected in the synthesis of high-quality and reliable sequences upon which the different rules are inferred. The quality of reconstruction is measured as a percentage of the number of reconstructed sequences with a certain number of reconstruction errors in the different regions to the total number of sequences, and the average number of reconstruction mistakes. It is clear that including the efficiency information increased the reconstruction quality, and decreased the average number of reconstruction mistakes. Nevertheless, there is on average 13.54 reconstruction mistakes in the sequences of length 34 . This is a source of some error, which can be analysed in the following results.

More concretely, Fig. 6 provides a pictorial agreement assessment between the generative CRISPR-VAE and the descriminative seq-deepCpf1 predictor. The figure shows the prediction of the seq-deepCpf 1 method on synthetic data claimed to belong to classes 0 -efficiency and 99-efficiency by CRISPR-VAE. Ideally, the figure should exhibit two disjoint peaks located at the two extremes of 0 and 99 . The figure indeed shows a tendency towards such behaviour, which can be quantified by a Spearman's correlation coefficient of $\sim 0.79$. In other words, according to seq-deepCpf1, most sequences claimed for class 99-efficiency have higher efficiencies than most sequences claimed for class 0 -efficiency, which enables the identification of the most prominent features that set the two types of sequences apart. It is worth pointing out that the reconstruction mistakes observed in table I are responsible for the small divergence from the ideal case as described.

\section{B. High-efficiency Features}

Herein, we include Fig. 7 for a holistic summary of the sequence-related features in different sequence regions and quadrants in the latent space. Said features pass the filtering stages and are significantly prominent in the sequences of class
TABLE I

COMPARISON BETWEEN CVAE AND VAE QUALITY OF RECONSTRUCTION

\begin{tabular}{|c|c|c|c|}
\cline { 2 - 4 } \multicolumn{1}{c|}{} & $\begin{array}{c}\text { mistakes } \leq \mathbf{2} \text { in } \\
\text { the seed region }\end{array}$ & $\begin{array}{c}\text { mistakes } \leq \mathbf{1 0} \\
\text { overall }\end{array}$ & $\begin{array}{c}\text { Average recon. } \\
\text { mistakes }\end{array}$ \\
\hline CVAE & $64 \%$ & $19 \%$ & 13.54 \\
\hline VAE & $50 \%$ & $0.2 \%$ & 16.86 \\
\hline
\end{tabular}

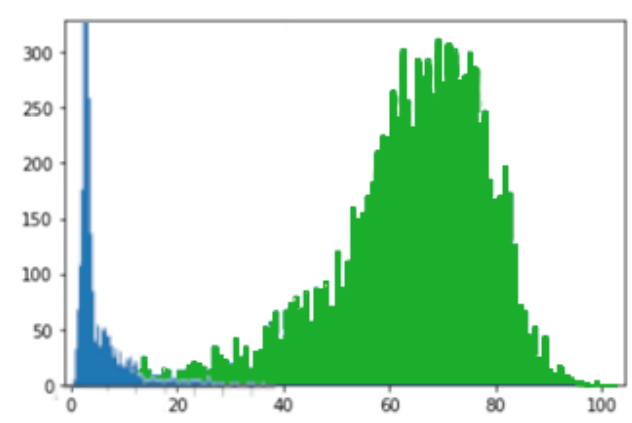

Fig. 6. seq-deepCpf1 [10] prediction on the synthetic data of efficiencies 0 (left) \& 99 (right)

99-efficiency in contrast to sequences of class 0 -efficiency. Filtering with the significance threshold results in focusing on the prominent features, and consequently having some empty sub-regions in Fig. $(7, \mathrm{c})$. The MSMs consist of cocentric significance circles, whose radii are proportional to their significance. The different regions are color-coded as per the legend in Fig. $(7, a)$. The discovered mers are scattered in the MSMs based on their significance and position in the gRNA; the larger the angle at which the mer is located, the more down the gRNA stream the mer feature exists. For clarity, we also segregated each region into three sub-regions separated by dash lines. These sub-regions describe the beginning, the middle, and the end of each region. In Fig. $(7, c)$, we highlight the significant features found in the synthetic data which are obscure in the training data HT1, but are confirmed by the testing data HT2 and HT3 by circling them.

Fig. 7 also provides a pictorial summary of the distinguishing trends that set the two types of sequences apart using CAM via a specialized classifier. The classifier learns to classify the synthetic data to a near-perfect degree with only few epochs (accuracy $\sim 95 \%$, with 5 epochs), alluding to how distinct the features in both categories are. We also include Fig. $(7, b)$ to show the benefit of segregating the analysis to different quadrants in the latent space. Otherwise, the analysis reveals an averaged version where only the globally prominent features are highlighted while ignoring many other valid ones. For example, the averaged summary in Fig. $(7, b)$ reveals a disfavoring of Thymine right after the PAM, which agrees with the existing findings [5], but does not reveal much more than that.

Many sequence-related features that result in high-efficiency sequences can be inferred by looking at Fig. 7. We focus especially on the features that are agreed upon by the two methods of CAMs and MSMs. Firstly, Adenine is preferred mainly in the promiscuous region, as shown in quadrants 1 and 3 (Q1 and Q3), as shown in the CAMs, and confirmed by some mers in the MSMs. Adenine therein especially prefers to 


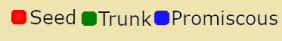

(a)
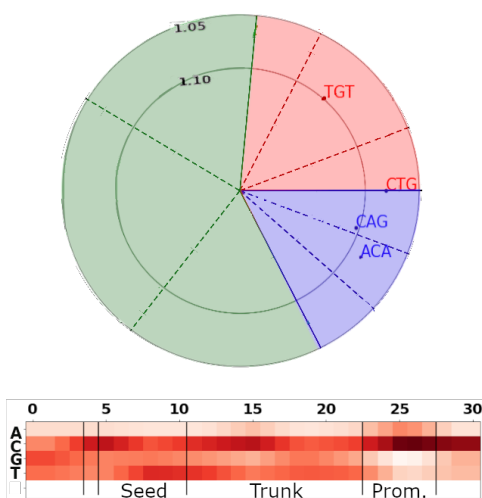

(b)
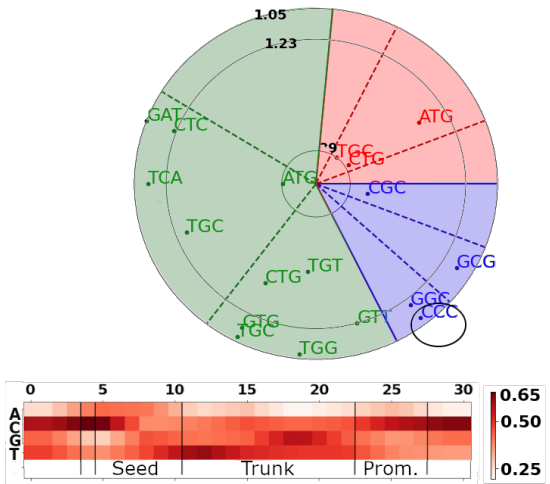

$<$
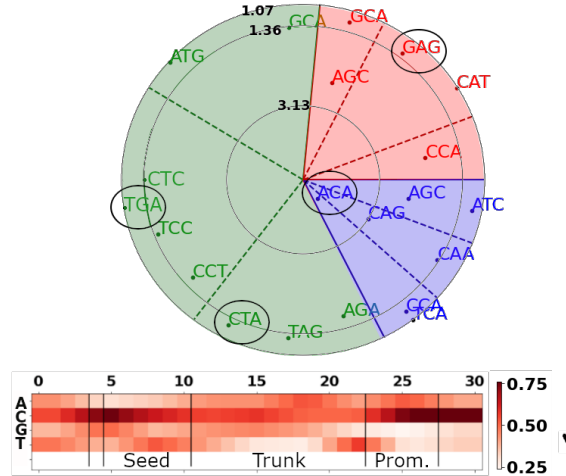

0.25

(c)
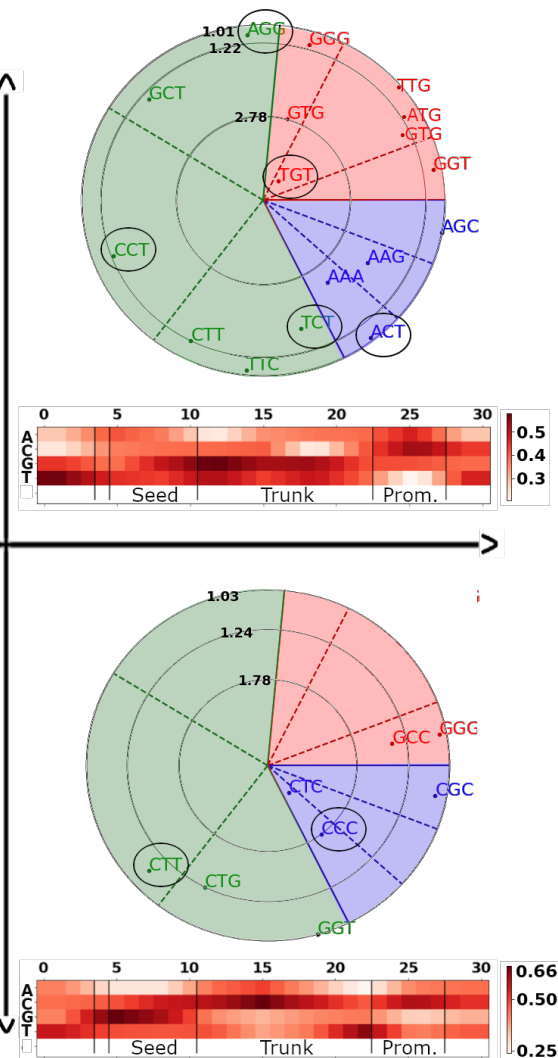

Fig. 7. Summary of high-efficiency sequence-related features as MSMs and CAMs, showing the benefit of a quadrant-based analysis (c), as compared to non-quadrant-based (b). The sequence regions are color-coded according to the legend in (a), and separated into three sub-regions (beginning $\rightarrow$ mid $\rightarrow$ end) going counter-clockwise; the circled mers in (c) can be exclusively found from the synthetic data, and not the training data, alluding to the added generality conferred by the proposed paradigm. In the MSMs, more significant features are closer to the center. Only the last nucleotide in the PAM is shown in the CAMs.

TABLE II

THE P-VALUES OF OBSERVING A SEQUENCE OF CLASS 99-EFFICIENCY; CALCULATED FROM A BINOMIAL DISTRIBUTION WITH BASE PROBABILITY OF $0.25^{3}$.

\begin{tabular}{|c|c|c|c|c|c|c|c|}
\hline \multicolumn{2}{|c|}{ Quadrant 1} & \multicolumn{2}{|c|}{ Quadrant 2} & \multicolumn{2}{|c|}{ Quadrant 3} & \multicolumn{2}{|c|}{ Quadrant 4} \\
\hline 3-mer & p-value & 3-mer & p-value & 3-mer & p-value & 3-mer & p-value \\
\hline S-beg: GGT & $1.35 \mathrm{E}-25$ & S-mid: CTG & $2.14 \mathrm{E}-59$ & S-beg: CCA & $1.37 \mathrm{E}-43$ & S-beg: GCC & $1.81 \mathrm{E}-38$ \\
\hline S-mid: ATG & $2.32 \mathrm{E}-30$ & S-mid: ATG & $1.07 \mathrm{E}-38$ & S-mid: CAT & $5.72 \mathrm{E}-19$ & S-beg: GGG & $5.86 \mathrm{E}-28$ \\
\hline S-mid: GTG & $1.06 \mathrm{E}-29$ & S-mid: TGC & $2.13 \mathrm{E}-60$ & S-mid: GAG & $1.35 \mathrm{E}-25$ & T-mid: CTT & $8.41 \mathrm{E}-31$ \\
\hline S-mid: TTG & $2.84 \mathrm{E}-23$ & T-mid: CTC & $3.04 \mathrm{E}-31$ & S-end: AGC & $5.87 \mathrm{E}-53$ & T-end: CTG & $2.84 \mathrm{E}-35$ \\
\hline S-mid: TGT & $5.80 \mathrm{E}-100$ & T-mid: GAT & $9.56 \mathrm{E}-25$ & S-end: GCA & $2.84 \mathrm{E}-23$ & T-end: GGT & $1.89 \mathrm{E}-26$ \\
\hline S-end: GTG & $3.09 \mathrm{E}-66$ & T-mid: ATG & $1.19 \mathrm{E}-60$ & T-beg: GCA & $3.56 \mathrm{E}-28$ & P-beg: CTC & $3.79 \mathrm{E}-60$ \\
\hline S-end: GGG & $5.86 \mathrm{E}-28$ & T-mid: TCA & $9.65 \mathrm{E}-28$ & T-beg: ATG & $5.72 \mathrm{E}-19$ & P-beg: CCC & $3.76 \mathrm{E}-47$ \\
\hline T-beg: AGG & $5.06 \mathrm{E}-26$ & T-mid: TGC & $2.28 \mathrm{E}-34$ & T-mid: CTC & $9.65 \mathrm{E}-28$ & P-end: CGC & $7.92 \mathrm{E}-29$ \\
\hline T-beg: GCT & $1.80 \mathrm{E}-33$ & T-end: CTG & $2.57 \mathrm{E}-40$ & T-mid: TGA & $8.94 \mathrm{E}-20$ & & \\
\hline T-mid: CCT & $5.06 \mathrm{E}-31$ & T-end: GTG & $3.56 \mathrm{E}-28$ & T-mid: TCC & $1.75 \mathrm{E}-29$ & & \\
\hline T-end: CTT & $3.85 \mathrm{E}-30$ & T-end: TGC & $8.28 \mathrm{E}-26$ & T-mid: CCT & $3.94 \mathrm{E}-32$ & & \\
\hline T-end: TTC & 4.11E-24 & T-end: TGT & $5.86 \mathrm{E}-46$ & T-end: CTA & $4.11 \mathrm{E}-24$ & & \\
\hline T-end: TCT & $5.16 \mathrm{E}-41$ & T-end: TGG & $8.28 \mathrm{E}-26$ & T-end: TAG & $8.28 \mathrm{E}-26$ & & \\
\hline P-beg: AAA & $3.70 \mathrm{E}-56$ & T-end: GTT & $6.57 \mathrm{E}-32$ & T-end: AGA & $1.80 \mathrm{E}-33$ & & \\
\hline P-beg: ACT & $1.75 \mathrm{E}-23$ & P-beg: GGC & $2.32 \mathrm{E}-30$ & P-beg: ACA & $3.54 \mathrm{E}-95$ & & \\
\hline P-mid: AAG & $1.37 \mathrm{E}-48$ & P-beg: CCC & $1.89 \mathrm{E}-26$ & P-beg: CCA & $4.59 \mathrm{E}-23$ & & \\
\hline P-end: AGC & $5.02 \mathrm{E}-22$ & P-mid: GCG & $2.16 \mathrm{E}-28$ & P-beg: TCA & $1.44 \mathrm{E}-18$ & & \\
\hline & & P-end: CGC & $1.16 \mathrm{E}-55$ & P-mid: CAG & $5.24 \mathrm{E}-67$ & & \\
\hline & & & & P-mid: CAA & $2.16 \mathrm{E}-28$ & & \\
\hline & & & & P-end: AGC & $5.57 \mathrm{E}-52$ & & \\
\hline & & & & P-end: ATC & $1.20 \mathrm{E}-22$ & & \\
\hline
\end{tabular}


combine with Cytosine or Guanine in said region (e.g., AAA and AAG in Q1, ACA and CAA in Q3).

As for Cytosine, it can be positioned everywhere in the gRNA, albeit in different combinations depending on the region, as revealed looking at the different quadrants. More concretely, for Cytosine in to be in the seed region, it prefers to combine with the TG pair (e.g., TGC and CTG in Q2), or preceded by Guanine (e.g., AGC, GCA in Q3), or followed by Adenine (e.g., GCA, CCA, CAT in Q3). As such, one can conclude that a motif of TGCA is observed in the seed region of efficient sequences. For Cytosine to be in the middle towards the end of the trunk region, it prefers to be combined with Thymine as observed in different mers in various quadrants. As for the promiscuous region, Cytosine prefers to either combine with Guanine (e.g., CGC, GCG in Q2) or be followed by Adenine (e.g., ACA, CAG, CAA, CCA, TCA in Q3).

As for Guanine, it can be placed at the beginning of the seed region if followed by Cytosine as revealed in Q4 (e.g., GCC), or anywhere in the seed region if preceded by Thymine or Adenine (e.g., TGT, TTG, GTG, ATG), or in the beginning of the trunk region (e.g., AGG), as revealed in Q1.

Thymine makes an appearance in various places. It prefers to combine with Cytosine in the middle towards the end of the trunk region as revealed in Q1, Q3, and Q4. For Thymine to be in the beginning towards the middle of the trunk region, it prefers to be followed by Guanine (e.g., TGT, TGG, TGC, GTG) as shown in Q2. Thymine is disfavored in the seed and promiscuous regions, except as auxiliary bases in a few cases.

The aforementioned features and many others can be inferred and summarized, particularly those that have been revealed exclusively by the synthetic data. In the MSMs, multiple such features are included, such as the TGT mer in the seed region, ACT, CCC, and ACA in the promiscuous region, and many other ones in the trunk region across the different quadrants. These features exist in the testing data HT2 and HT3, but are obscurely observed in the training data HT1. This showcases the direct benefit of the suggested paradigm, where it is possible to discover obscure features that lie in the neighborhood of the prominent ones of the training data.

Moreover, table II provides the results of testing the statistical significance of the mers using Welch's t-test. Naturally, the table agrees with the MSMs, showing larger values for mers closer to the center. Also, all features demonstrate extremely small numbers ( $p$-value $\ll 0.05$ ), since the suggested significance threshold in (5) is more stringent than the threshold on the randomness assumption in the hypothesis testing.

\section{Controlled Sequence Generation}

Finally, an additional benefit of the quadrant-based analysis is that it enables low-level editing control in the sequence generation. More concretely, the decoder shown in Fig. 2 is a standalone, efficiency-aware sequence generator. The user inputs in the efficiency score channel the desired efficiency, and feeds in the latent code channel a $2 \mathrm{D}$ code from the quadrant which exhibits certain wanted features.

For example, latent codes sampled from the right side of the latent space (Q1, and Q4) exhibit more Guanine content in the

\begin{tabular}{|c|c|}
\hline Code & Sequence \\
\hline$(0.59,0.59)$ & TACC T T T TGG T G T GCA TA G T C T T T T T:A C T G T G C T \\
\hline$(-0.59,0.59)$ & 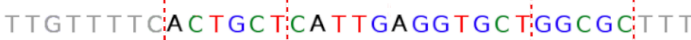 \\
\hline$(-0.59,-0.59)$ & CGTCTTTAG CATGCTGATCGACGA GA:A CA G C C TC \\
\hline \multirow[t]{2}{*}{$(0.59,-0.59)$} & T T GT T T T G G C GCA T:G TCT T C CA TC T T:C TC TA:G T \\
\hline & Promiscuous \\
\hline
\end{tabular}

Fig. 8. The coordinates of the centroid of each quadrant decoded into a gRNA sequence

seed region. Traversing to the left (Q2), the Guanine content in the seed region decreases, and the Cytosine content increases. Between Q1 and Q4, the former should be sampled from for a seed region that has Guanine and Thymine, and the latter for one that has Guanine and Cytosine. To control the beginning of the trunk region, the maps in Fig. 7 reveal that Guanine and Cytosine contents can be controlled as per the following. For sequences with Guanine-rich in the beginning of the trunk region, Q1 must be sampled from. Traversing downwards (Q4) is the direction of the most increase in Cytosine and decrease in Guanine; traversing left $(\mathrm{Q} 2)$ has a similar effect but in a lesser magnitude. As for the middle of the trunk region, codes must be taken from Q4 for more Cytosine and Thymine, and from Q2, for less Cytosine and more Guanine. Additionally, more Adenine in the end of the trunk region can be obtained by inputting codes from Q3. In the same region (trunk-end), the Thymine content can be increased by sampling from Q1 (and to a lesser degree from Q4), and the Thymine-Guanine pair can be increased by sampling from Q2. Lastly, for more Adenine in the promiscuous region, Q1 or Q3 must be sampled; the latter emphasizes the Cytosine combination with Adenine. For more Guanine-Cytosine in the promiscuous region, codes must be taken from Q2.

In Fig. 8, we show the generated sequences that correspond to 4 latent codes, each collected from a quadrant; specifically, the coordinates of the centroid of each quadrant, as shown in Fig. 1. In our analysis, the codes sampled from the latent space are normally distributed, expanding from $(-1.64,-1.64)$ to $(1.64,1.64)$. Many of the above-mentioned traits can be conspicuously seen in such sequences. This confirms the low-level control capability of CRISPR-VAE as a sequence generator.

\section{CONCLUSIONS}

In this paper, we developed a complete paradigm towards improving the explainability of deep learning-based models in the application of gRNA sequence efficiency prediction in CRISPR systems. The paradigm consists of building a generative framework where synthetic data is generated that resembles labeled training data, and fills in the sequencerelated gaps in it. The agreement between the proposed generative framework and the descriminative seq-DeepCpf 1 increases the confidence in the findings, and also provides explainability for the decision of the descriminative method. Two analysis methods were used to infer and summarize the most prominent features from the synthetic data. The first is a manual histogramming method, and the second is automatic, using class 
activation maps. Many features have been thus discovered and highlighted, including particularly obscure ones that are confirmed to be in the testing data. Lastly, we showcased the capability of the proposed framework in generating gRNA sequences, with a low-level editing control, by altering the latent code. We further mapped out the relationship between the position of the latent code and the expected features in the generated sequence.

\section{FUNDING}

This work was supported by the Abu Dhabi Award for Research Excellence under ASPIRE/Advanced Technology Research Council.

\section{REFERENCES}

[1] M. Adli, "The crispr tool kit for genome editing and beyond," Nature communications, vol. 9, no. 1, pp. 1-13, 2018.

[2] Y. Wu, D. Liang, Y. Wang, M. Bai, W. Tang, S. Bao, Z. Yan, D. Li, and $\mathrm{J}$. Li, "Correction of a genetic disease in mouse via use of crispr-cas9," Cell stem cell, vol. 13, no. 6, pp. 659-662, 2013.

[3] R. J. Platt, S. Chen, Y. Zhou, M. J. Yim, L. Swiech, H. R. Kempton, J. E. Dahlman, O. Parnas, T. M. Eisenhaure, M. Jovanovic et al., "Crispr-cas9 knockin mice for genome editing and cancer modeling," Cell, vol. 159 , no. 2, pp. 440-455, 2014

[4] L. Cong, F. A. Ran, D. Cox, S. Lin, R. Barretto, N. Habib, P. D. Hsu, $\mathrm{X}$. Wu, W. Jiang, L. A. Marraffini et al., "Multiplex genome engineering using crispr/cas systems," Science, vol. 339, no. 6121, pp. 819-823, 2013.

[5] H. Zhu and C. Liang, "Crispr-dt: designing grnas for the crispr-cpf1 system with improved target efficiency and specificity," Bioinformatics, vol. 35, no. 16, pp. 2783-2789, 2019.

[6] A. Alok, D. Sandhya, P. Jogam, V. Rodrigues, K. K. Bhati, H. Sharma, and J. Kumar, "The rise of the crispr/cpf 1 system for efficient genome editing in plants," Frontiers in Plant Science, vol. 11, p. 264, 2020. [Online]. Available: https://www.frontiersin.org/article/10.3389/fpls.2020.00264

[7] G. Zhang, Z. Dai, and X. Dai, "C-rnncrispr: Prediction of crispr/cas9 sgrna activity using convolutional and recurrent neural networks," Computational and structural biotechnology journal, vol. 18, pp. 344-354, 2020.

[8] H. Xu, T. Xiao, C.-H. Chen, W. Li, C. A. Meyer, Q. Wu, D. Wu, L. Cong, F. Zhang, J. S. Liu et al., "Sequence determinants of improved crispr sgrna design," Genome research, vol. 25, no. 8, pp. 1147-1157, 2015.

[9] H. K. Kim, M. Song, J. Lee, A. V. Menon, S. Jung, Y.-M. Kang, J. W. Choi, E. Woo, H. C. Koh, J.-W. Nam et al., "In vivo high-throughput profiling of crispr-cpf1 activity," Nature methods, vol. 14, no. 2, pp. 153-159, 2017.

[10] H. K. Kim, S. Min, M. Song, S. Jung, J. W. Choi, Y. Kim, S. Lee, S. Yoon, and H. H. Kim, "Deep learning improves prediction of crisprcpf1 guide rna activity," Nature biotechnology, vol. 36 , no. 3, p. 239, 2018.

[11] G. Zhang and X. Dai, "Cnn-svr for crispr-cpf1 guide rna activity prediction with data augmentation," in Proceedings of the 2019 9th International Conference on Bioscience, Biochemistry and Bioinformatics, 2019, pp. 43-47.

[12] G. Chuai, H. Ma, J. Yan, M. Chen, N. Hong, D. Xue, C. Zhou, C. Zhu, K. Chen, B. Duan et al., "Deepcrispr: optimized crispr guide rna design by deep learning," Genome biology, vol. 19, no. 1, pp. 1-18, 2018.

[13] L. Xue, B. Tang, W. Chen, and J. Luo, "Prediction of crispr sgrna activity using a deep convolutional neural network," Journal of chemical information and modeling, vol. 59, no. 1, pp. 615-624, 2018.

[14] H. K. Kim, G. Yu, J. Park, S. Min, S. Lee, S. Yoon, and H. H. Kim, "Predicting the efficiency of prime editing guide rnas in human cells," Nature Biotechnology, vol. 39, no. 2, pp. 198-206, 2021.

[15] Q. Liu, X. Cheng, G. Liu, B. Li, and X. Liu, "Deep learning improves the ability of sgrna off-target propensity prediction," BMC bioinformatics, vol. 21 , no. 1, pp. 1-15, 2020.

[16] J. Lin and K.-C. Wong, "Off-target predictions in crispr-cas9 gene editing using deep learning," Bioinformatics, vol. 34, no. 17, pp. i656i663, 2018.
[17] J. Charlier, R. Nadon, and V. Makarenkov, "Accurate deep learning offtarget prediction with novel sgrna-dna sequence encoding in crispr-cas9 gene editing," Bioinformatics (Oxford, England), p. btab112, 2021.

[18] G. Zhang, T. Zeng, Z. Dai, and X. Dai, "Prediction of crispr/cas9 single guide rna cleavage efficiency and specificity by attention-based convolutional neural networks," Computational and structural biotechnology journal, vol. 19, pp. 1445-1457, 2021.

[19] A. R. O'Brien, G. Burgio, and D. C. Bauer, "Domain-specific introduction to machine learning terminology, pitfalls and opportunities in crispr-based gene editing," Briefings in bioinformatics, vol. 22, no. 1, pp. 308-314, 2021.

[20] X. Wang, X. Wang, R. K. Varma, L. Beauchamp, S. Magdaleno, and T. J. Sendera, "Selection of hyperfunctional sirnas with improved potency and specificity," Nucleic acids research, vol. 37, no. 22, pp. e152-e152, 2009.

[21] N. Wong, W. Liu, and X. Wang, "Wu-crispr: characteristics of functional guide rnas for the crispr/cas9 system," Genome biology, vol. 16, no. 1, pp. 1-8, 2015.

[22] K. Sohn, H. Lee, and X. Yan, "Learning structured output representation using deep conditional generative models," Advances in neural information processing systems, vol. 28, pp. 3483-3491, 2015.

[23] B. Zhou, A. Khosla, A. Lapedriza, A. Oliva, and A. Torralba, "Learning deep features for discriminative localization," in Proceedings of the IEEE conference on computer vision and pattern recognition, 2016, pp. 29212929. 